\title{
Does the Internet promote the unregulated use of fecal microbiota transplantation: a potential public health issue?
}

This article was published in the following Dove Press journal: Clinical and Experimental Gastroenterology

\author{
Jonathan Philip Segal' \\ Faisal Abbasi \\ Cynthia Kanagasundaram ${ }^{3}$ \\ Ailsa Hart' \\ 'Department of Gastroenterology, St \\ Mark's Hospital, Harrow, UK, ${ }^{2}$ Lister \\ Hospital, Stevenage, UK, ${ }^{3}$ Department \\ of Gastroenterology, Addenbrooke's \\ Hospital, Cambridge, UK
}

\begin{abstract}
Introduction: The Internet has become an increasingly popular resource for medical information. Fecal microbiota transplantation (FMT) has changed the treatment of Clostridium difficile with cure rates of $81 \%$ following one infusion of FMT, further studies have since validated these findings. The Medicines and Health care Products Regulatory Agency has classified FMT as a medicine and hence should be only utilized in strict clinical settings.

Methods: We searched Facebook, Twitter, Google, and YouTube using the words "Faecal Microbiota Transplantation" and "FMT". We utilized the first 50 hits on each site. We analyzed the percentage of articles that fell outside regulated medical practice. We searched how many clinics in the UK advertised practice that falls outside suggested guidelines.

Results: Google, YouTube, and Facebook had a variety of information regarding FMT available. Nine out of 50 (18\%) of the top 50 google searches can be considered articles that fall outside regulated practice. YouTube highlighted four videos describing how to self-administer FMT, one of these was for ulcerative colitis. Fourteen percent of the top 50 YouTube videos fall outside regulated practice and $8 \%$ of the top 50 Facebook searches fall outside regulated clinical practice. There were two clinics in the UK advertising FMT for uses that fall outside regulated practice. Conclusion: Clinicians and patients need to be aware of the resources available through social media and the Internet. It should be appreciated that some websites fall outside regulated clinical practice. Private clinics offering FMT need to ensure that they are offering FMT within a regulated framework.
\end{abstract}

Keywords: fecal microbiota transplantation, FMT, microbiota, Internet, social media

\section{Background}

The use of the Internet has become an increasingly popular resource for medical information. There is an estimated 3.5 billion Internet users worldwide. ${ }^{1}$ Facebook has 1.71 billion monthly active users ${ }^{2}$ and Twitter has 313 million monthly active users. ${ }^{3}$ Various investigators have critically evaluated websites and patient-oriented medical information on the Internet and found them to be scientifically inaccurate and incomplete. ${ }^{4-8}$ While quality of information has been regarded as poor, other authors have found that the use of the Internet can have a positive impact on patient knowledge. ${ }^{9}$ The three main ways of accessing online health information are: 1) searching for health information; 2) participating in support groups; and 3) interacting with health professionals. ${ }^{10}$

Fecal microbiota transplantation (FMT) was first documented in the fourth century in China, where it was known as "yellow soup". It was used to treat diarrhea and food poisoning with documented good results. ${ }^{11}$ More recently, FMT has changed the
Correspondence: Jonathan Philip Segal St Mark's Hospital, IBD Unit, Watford Road, Harrow HAI 3UJ, UK Tel +4407809l48950 Email jonathansegal@doctors.org.uk 
treatment of refractory Clostridium difficile diarrhea with cure rates of $81 \%$ following one infusion of FMT. ${ }^{12}$ Further studies have since validated these findings. ${ }^{13}$

Despite its success, regulated uptake of the use of FMT is still sparse in the UK as demonstrated by a national survey, which suggested that only $28 \%$ of the UK hospitals use FMT. $^{14}$

With the success of FMT for $C$. difficile, it has been used for research purposes for many other conditions, including psychiatric, ${ }^{15}$ Parkinson's disease,${ }^{16}$ metabolic disorders, ${ }^{17}$ and inflammatory bowel diseases. ${ }^{18}$

Despite the success of FMT, only short-term safety has been assessed. ${ }^{19}$ Long-term safety outcomes are not yet available. A systematic review has highlighted that FMT can be associated with significant adverse events. ${ }^{20}$ More recent data has suggested that phenotypes can also be transferred by FMT such as obesity. ${ }^{21}$

In view of the relative deficiencies in our knowledge of safety of FMT, The Medicines and Health care Products Regulatory Agency has now classified FMT as a medicine and hence should be only utilized in strict clinical settings. ${ }^{22}$ It is currently only licensed for the treatment of refractory C. difficile infection, and guidelines outlining its safe clinical use are available to try and minimize potential risk to patients. ${ }^{19}$

Despite the strict governance of FMT, there is evidence that FMT is still being used outside of licensed practice with "DIY" websites, ${ }^{23}$ and clinics offering FMT for conditions other than $C$. difficile infection. This research will review the online material available regarding FMT and explore how much information falls outside licensed practice and is, therefore, potentially harmful for the public.

Our study aims to search Facebook, Twitter, Google, and YouTube to assess the material available on FMT and to calculate the percentage articles that fall outside regulated practice. We also looked to search how many private clinics in the UK are offering FMT that fall outside suggested clinical practice.

\section{Materials and methods}

This was a cross-sectional online analysis study of information on fecal microbiota transplantation on the Internet. We searched Facebook, Twitter, Google, and YouTube using the words "Faecal Microbiota Transplantation" and "FMT". We looked through the first 50 hits on each site. The search was performed on May 1, 2017. We restricted the search to the top 50 articles on each platform. This was chosen as we thought it would represent the top 50 articles in terms of popularity and reviews. It is therefore likely that the top 50 articles represent those that are seen by the target audiences.

We categorized the results into:

1. Education material by medical professions, which was subdivided into the following:

- Generic hospital and clinic information

- Information from scientific journals/books

- Communication between health care professionals to patients

2. Education material by another group

3. Charity

4. Support group

5. News article

6. Self-administration group

7. Medical professional clinic

8. Humorous

A sub-analysis of Google will be used to search for private clinics offering FMT. Content of the clinics webpage will be analyzed for indications and cost of FMT.

\section{Bias}

The Internet is a source of bias, and quality and accuracy of information is very difficult to assess online. To try and objectively analyze bias, we have cross-referenced the online information against the regulated clinical use of FMT.

\section{Results Google search}

There were 51,000 hits when searching Google (Figure 1). The top 50 search terms using Google had 13 different categories of information. Seventeen of the top 50 search terms were scientific journals. Eight articles were news articles. Five articles were from regulatory bodies and five were educational material from health care professionals. There were three articles relating to self-administration of FMT with one article relating to a private clinic offering FMT. Twenty-eight articles were related to $C$. difficile, ten articles were related to all uses of FMT, four articles were related to ulcerative colitis, four articles were related to IBD, three articles did not specify the use of FMT, and one was for insulin resistance. Nine out of $20(18 \%)$ of the top 50 Google searches can be considered articles that fall outside regulated practice. 


\section{Private clinic search using Google}

Using the search query "FMT clinics" in Google, there were 92,700 hits. Forty-nine of these were either direct links to or indirectly linking to a UK private clinic. The clinic provided a full treatment schedule for $£ 3700$, which includes an initial consultation followed by ten in-house treatments, two takehome treatments, and a follow-up consultation 3 months posttreatment. They advertise FMT treatment for a variety of conditions, including inflammatory bowel disease, multiple sclerosis, and Parkinson's disease. The single other top 50 hit referred to another UK clinic; however, the website gave no indication of treatment regime or cost, and this information was not available upon telephoning the clinic contact number.

\section{YouTube search}

There were 2930 total hits when searching YouTube (Figure 2). Videos originated from 2013 to 2016, with viewings ranging from five views to $1,100,000$ views. Using the top 50 search videos on YouTube, 13 videos were information to patients from health care professionals, 12 videos were lectures for health care professionals, six were news articles, five were patients' own experiences of FMT with three selfadministration videos. There were three private medical clinics offering FMT; of these, two were UK private clinics, which advertised FMT that fall outside regulated clinical practice. Twenty-four videos were about the use of FMT for $C$. difficile diarrhea, with seven videos based on information regarding

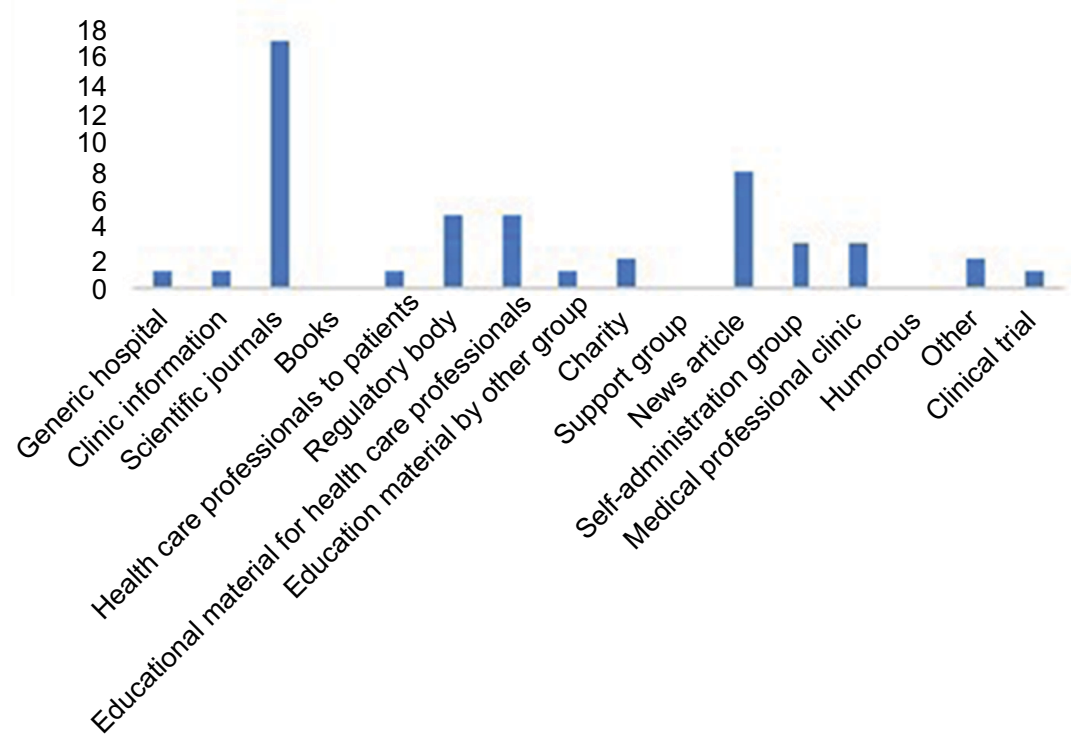

Figure I Google search.

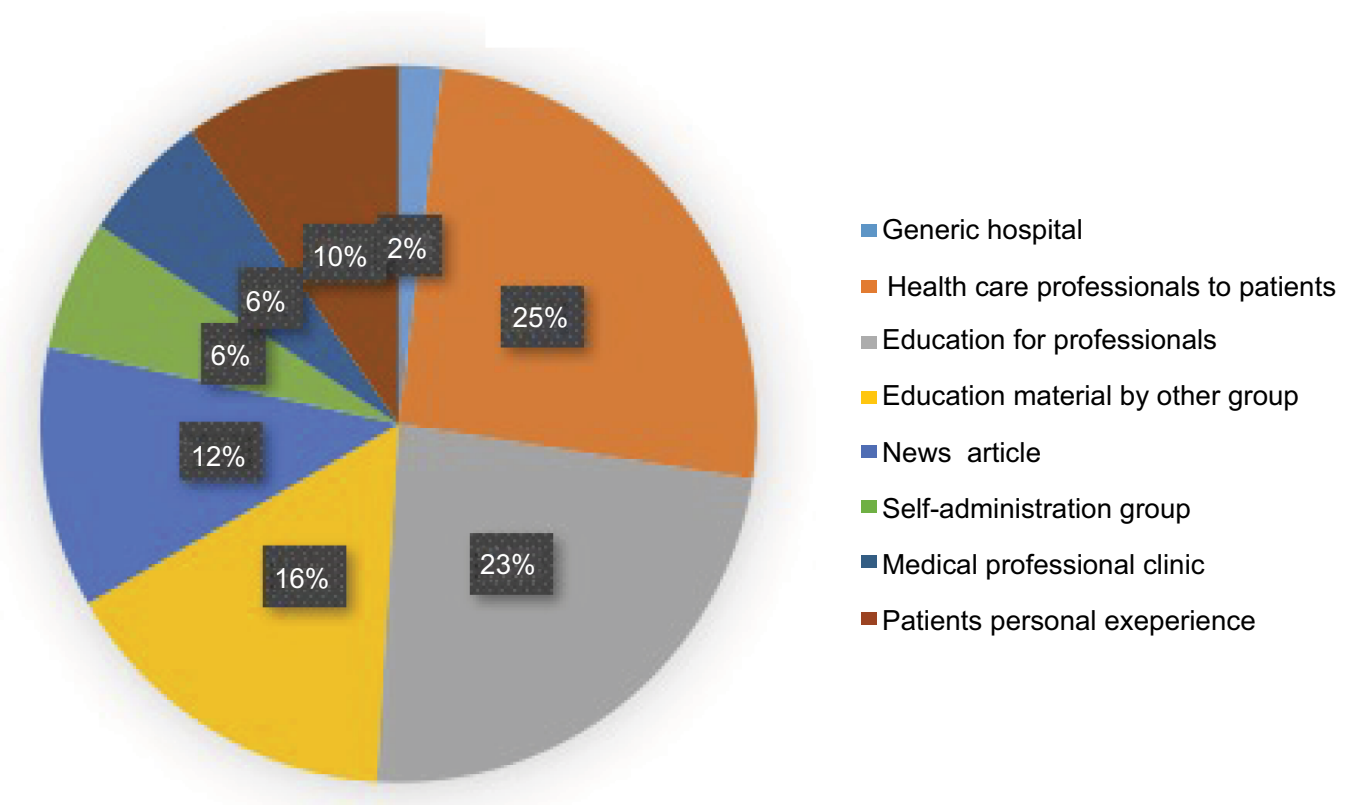

Figure 2 YouTube search. 
ulcerative colitis. Four videos were based on all indications for FMT, 14 videos did not indicate specific indication. One video was for the use of FMT for irritable bowel syndrome. The self-administration videos were viewed 154,900 times, one self-administration video was for the treatment of ulcerative colitis, and three videos did not give any indication. Seven out of 50 videos (14\%) fall outside regulated practice.

\section{Facebook search}

Of the top 50 Facebook searches, 19/50 were links to scientific journals, 12 were links to news articles, seven articles were educational material by a non-scientific group, six articles were educational materials based for professionals, three articles were educational material for patients, with one link to a charity, and one link to a support group. Eighteen articles did not mention an indication for FMT. Seventeen articles were related to the treatment of $C$. difficile, eleven articles were for all uses of FMT. Four articles were for inflammatory bowel disease. Four out of 50 (8\%) Facebook searches can be considered articles that fall outside regulated practice.

\section{Twitter search}

There were zero results using the search query "Faecal microbiota transplantation".

\section{Discussion}

This study has highlighted that some potentially unregulated practice regarding FMT is easily available through private clinics and Internet resources. Long-term follow-up beyond 5 years is lacking on the safety of FMT and therefore its use should be carefully regulated to avoid potential unknown complications. ${ }^{24-26}$ This study is the first to our knowledge to highlight that the Internet can promote the unregulated use of FMT and something that both clinicians and patients should be aware of.

This study has highlighted that there are variable sources of information available to patients. Eighteen percent of the top 50 Google searches can be considered articles that fall outside regulated practice, with $14 \%$ of the top 50 YouTube videos and $8 \%$ of the top 50 Facebook searches falling outside regulated clinical practice. Most alarmingly, FMT is still being advertised through private clinics that are using it for indications that have not been licensed. There are still many resources where patients can learn how to self-administer FMT. Most other sources of Internet information are mostly educational material for health care professionals and patients.

Social media is a developing and popular tool for patients to gather information. As technology advances, social media will be used more to provide patients and clinicians with information at the click of a button. It is therefore essential that physicians educate patients on ways of obtaining accurate information, while warning against some of the lower quality evidence available.

There is a vast amount of information regarding FMT available through the Internet and social media. The quality and accuracy of Internet information has been previously reported as very variable. ${ }^{27}$ As FMT in the UK is now considered a medical therapy, strict regulation needs to be applied. Long-term safety effects of FMT are yet to be fully understood. It is therefore possible that potential recipients of FMT may come to harm by using the Internet resources available.

There are limitations with this study. The quality of information available on the Internet was not formally assessed for accuracy. It is unknown how many people use the Internet advice to guide their treatment plans.

The Internet is difficult to monitor for correct and accurate information. In terms of clinical utility of FMT, future work should ensure those that offer FMT as a treatment are doing so under a regulated license for approved indications.

\section{Conclusion}

There is a vast amount of information available about FMT through social media that has the potential for causing harm. Private clinics offering FMT need to ensure that they are offering FMT within a regulated framework. A collaborative approach between clinicians, public health bodies, and regulators of social media should help block potentially dangerous sources of information on social media platforms.

\section{Data sharing statement}

All data is freely available through search on social media, which includes Google, Facebook, Twitter, and Youtube. The datasets used and/or analyzed during the current study are available from the corresponding author on request.

\section{Acknowledgments}

All authors have agreed to the content of the manuscript and consent to publication. This work was presented in abstract format at the European Crohn's and Colitis meeting in Vienna, Austria, on February 16, 2017.

\section{Author contributions}

All authors made substantial contributions to conception and design, acquisition of data, or analysis and interpretation of data; took part in drafting the article or revising it critically 
for important intellectual content; gave final approval of the version to be published; and agree to be accountable for all aspects of the work.

\section{Disclosure}

The authors report no conflicts of interest in this work.

\section{References}

1. Number of Internet Users (2016) - Internet Live Stats. Available from: http://www.internetlivestats.com/internet-users/. Accessed May 1, 2017.

2. Facebook users worldwide 2016|Statista. Available from: https:// www.statista.com/statistics/264810/number-of-monthly-activefacebook-users-worldwide/. Accessed May 1, 2017.

3. Twitter: monthly active users 2010-2016 - Statista. Available from: https://www.statista.com/statistics/282087/number-of-monthly-activetwitter-users/. Accessed May 1, 2017.

4. Al-Bahrani A, Plusa S. The quality of patient-orientated Internet information on colorectal cancer. Color Dis. 2004;6(5):323-326.

5. Beredjiklian PK, Bozentka DJ, Steinberg DR, Bernstein J. Evaluating the source and content of orthopaedic information on the Internet. The case of carpal tunnel syndrome. J Bone Joint Surg Am. 2000;82-A(11): 1540-1543.

6. Sutherland LA, Wildemuth B, Campbell MK, Haines PS. Unraveling the web: an evaluation of the content quality, usability, and readability of nutrition web sites. J Nutr Educ Behav. 2005;37(6):300-305.

7. Morr S, Shanti N, Carrer A, Kubeck J, Gerling MC. Quality of information concerning cervical disc herniation on the Internet. Spine $J$. 2010;10(4):350-354.

8. Yeung TM, Mortensen NJ. Assessment of the quality of patientorientated Internet information on surgery for diverticular disease. Dis Colon Rectum. 2012;55(1):85-89.

9. Kothari M, Moolani S. Reliability of "Google" for obtaining medical information. Indian J Ophthalmol. 2015;63(3):267-269.

10. Cline RJ, Haynes KM. Consumer health information seeking on the Internet: the state of the art. Health Educ Res. 2001;16(6):671-692.

11. Zhang F, Luo W, Shi Y, Fan Z, Ji G. Should we standardize the 1,700-year-old fecal microbiota transplantation? Am J Gastroenterol. 2012;107(11): 1755 .

12. van Nood E, Vrieze A, Nieuwdorp M, et al. Duodenal infusion of donor feces for recurrent Clostridium difficile. $N$ Engl J Med. 2013;368(5): 407-415.
13. Ianiro G, Bibbò S, Scaldaferri F, Gasbarrini A, Cammarota G. Fecal microbiota transplantation in inflammatory bowel disease: beyond the excitement. Medicine (Baltimore). 2014;93(19):e97.

14. Quraishi MN, Segal J, Mullish B, et al. National survey of practice of faecal microbiota transplantation for Clostridium difficile infection in the UK. $J$ Hosp Infect. 2017;95(4):444-445.

15. Evrensel A, Ceylan ME. Fecal microbiota transplantation and its usage in neuropsychiatric disorders. Clin Psychopharmacol Neurosci. 2016;14(3):231-237.

16. Xu MQ, Cao HL, Wang WQ, et al. Fecal microbiota transplantation broadening its application beyond intestinal disorders. World J Gastroenterol. 2015;21(1):102-111.

17. Marotz CA, Zarrinpar A. Treating obesity and metabolic syndrome with fecal microbiota transplantation. Yale J Biol Med. 2016;89(3):383-388.

18. Paramsothy S, Paramsothy R, Rubin DT, et al. Faecal microbiota transplantation for inflammatory bowel disease: a systematic review and meta-analysis. J Crohns Colitis. 2017;11(10):1180-1199.

19. Cammarota G, Ianiro G, Tilg H, et al; European FMT Working Group. European consensus conference on faecal microbiota transplantation in clinical practice. Gut. 2017;66(4):569-580.

20. Wang S, Xu M, Wang W, et al. Systematic review: adverse events of fecal microbiota transplantation. PLoS One. 2016;11(8):e0161174.

21. Alang N, Kelly CR. Weight gain after fecal microbiota transplantation. Open Forum Infect Dis. 2015;2(1):ofv004.

22. Published MHRA and HTA guideline on FMT|General|Clinical Guidance. Available from: http://www.bsg.org.uk/clinical-guidance/general/ published-mhra-and-hta-guideline-on-fmt.html. Accessed May 1, 2017.

23. Fecal Transplant At Home - DIY Instructions|The Power of Poop. Available from: http://thepowerofpoop.com/epatients/fecaltransplant-instructions/. Accessed May 1, 2017.

24. Agrawal M, Aroniadis OC, Brandt LJ, et al. The long-term efficacy and safety of fecal microbiota transplant for recurrent, severe, and complicated Clostridium difficile infection in 146 elderly individuals. J Clin Gastroenterol. 2015;50(5):403-407.

25. Aroniadis OC, Brandt LJ, Greenberg A, et al. Long-term follow-up study of fecal microbiota transplantation (FMT) for severe or complicated Clostridium difficile infection (CDI). Gastroenterology. 2013; 144(5):S185.

26. Mamo Y, Woodworth M, Sitchenko K, Dhere T, Kraft CS. Durability and long-term clinical outcomes of Fecal Microbiota Transplant (FMT) treatment in patients with recurrent Clostridium difficile infection. Clin Infect Dis. Epub 2017 Dec 19.

27. Benigeri M, Pluye P. Shortcomings of health information on the Internet. Health Promot Int. 2003;18(4):381-386.

\section{Clinical and Experimental Gastroenterology}

\section{Publish your work in this journal}

Clinical and Experimental Gastroenterology is an international, peerreviewed, open access, online journal publishing original research, reports, editorials, reviews and commentaries on all aspects of gastroenterology in the clinic and laboratory. This journal is included on PubMed. The manuscript management system is completely online

\section{Dovepress}

and includes a very quick and fair peer-review system, which is all easy to use. Visit http://www.dovepress.com/testimonials.php to read real quotes from published authors. 